\title{
Diverse sapovirus genotypes identified in children hospitalised with gastroenteritis in selected regions of South Africa
}

Tanya Y Murray ${ }^{a}$, Sandrama Nadan ${ }^{a, b}$, Nicola A Page ${ }^{b}$ and Maureen B Taylor ${ }^{a}$

a.

Department of Medical Virology, Faculty of Health Sciences, University of Pretoria, Private Bag X323,

Arcadia, 0007, Pretoria, South Africa. Email: tanyaymurray@gmail.com (TYM);

maureen.taylor@up.ac.za (MBT)

b. Centre for Enteric Diseases, National Institute for Communicable Diseases, Private Bag X4,

Sandringham, 2131, Johannesburg, South Africa. Email: sandran@nicd.ac.za (SN);

nicolap@nicd.ac.za (NAP)

Correspondence:

Tanya Y Murray

Department of Medical Virology

University of Pretoria

Private Bag X323

Arcadia

Pretoria 0007

South Africa

Tel: +27123192534

Fax: +27123255550

Email: tanyaymurray@gmail.com

Abstract word count: 158 words

Text word count: 2512 words 


\section{Abstract:}

Background: Sapoviruses (SaVs) are recognised as causative agents of gastroenteritis worldwide. However, data on the genetic diversity of this virus in Africa is lacking, particularly in the form of current long-term studies.

Objective: To determine the genetic diversity of SaVs in children hospitalised with gastroenteritis in South Africa (SA).

Study design: From April 2009 to December 2013, SaVs were characterised from stool specimens from children hospitalised with gastroenteritis in four provinces of SA.

Results: Fourteen different SaV genotypes were identified from the 221 strains that were characterised. Genogroup (G) IV predominated overall and was detected in 24\% (53/221) of specimens. The other identified genotypes included six belonging to $\mathrm{GI}$ (GI.1, GI.2, GI.3, GI.5, GI.6, and GI.7) and seven belonging to GII (GII.1, GII.2, GII.3, GII.4, GII.5, GII.6 and GII.7).

Conclusion: This study has provided the first comprehensive data on the genetic diversity of SaVs in a clinical setting in SA, contributing to the global knowledge of this virus.

Keywords: Genotyping, molecular diversity, paediatric, sapovirus, South Africa

Abbreviations: Adenovirus, AdV; Doctor George Mukhari hospital, DGM; Genotype, G; Gauteng, GP; KwaZulu Natal, KZN; Mpumalanga, MP; Norovirus, NoV; Polymerase Chain Reaction, PCR; Rotavirus, RV; Rotavirus Sentinel Surveillance Programme, RSSP; South Africa, SA; Sapovirus, SaV; Western Cape, WC. 


\section{Background}

Sapovirus (SaV), a member of the Caliciviridae family, is a causative agent of gastroenteritis [1]. The virus is predominantly reported in outbreaks and sporadic cases of gastroenteritis in children [2-6], but has more recently been reported in adult populations [7-10]. Sapoviruses are small, non-enveloped, single-stranded RNA viruses. The genome is $7.3-7.5 \mathrm{~kb}$ in length and is arranged in two or three open reading frames [11]. The SaV genus is divided into at least five genogroups (GI-GV) based on the capsid gene sequence, of which GI, GII, GIV and GV include strains that infect humans [12]. Genogroups I and II are each further divided into seven genotypes [13].

There have been few long-term studies on the genetic diversity of SaVs in children with sporadic acute gastroenteritis. Currently the largest study, spanning nine years (2002-2011), has originated from Japan where SaVs were analysed in outpatients with gastroenteritis and GIV predominated until 2007 after which it was replaced by GII.3 [4]. In a two-year (2005-2007) study in Denmark, SaVs were characterised from children with gastroenteritis referred by general practitioners and GI.1 predominated [2]. These studies did not focus primarily on hospitalised children with severe gastroenteritis and even fewer studies on SaVs have been conducted in Africa. A recent study (2009-2010) from Burkina Faso reported nine SaV genotypes in children with gastroenteritis, with infections generally associated with milder symptoms [14]. In Tunisia, SaVs were reported in paediatric outpatients with gastroenteritis and only GI.1 was identified [15]. Sapoviruses have also been reported in patients hospitalised with gastroenteritis in Tanzania [16] and in Malawi [17]. Genotypic characterisation was not conducted in the Tanzanian study [16] and in Malawi, only GII strains were identified [17]. Five different SaV genotypes were identified in HIV-seropositive children in Kenya [18]. In South Africa (SA), SaVs were first reported in 1997 in $0.4 \%$ of patients with sporadic gastroenteritis [19]. In 2008, SaVs were reported in 4\% (10/245) of specimens from children hospitalised with gastroenteritis in the Pretoria region of Gauteng (GP), SA, but the strains were not further characterised [20]. 
There are no recent comprehensive studies on SaVs in southern Africa. Many studies on SaVs span less than two years and are restricted to one geographical region of a country. This may influence the diversity of genotypes identified.

\section{Objectives}

To determine the genetic diversity of SaVs in children hospitalised with gastroenteritis over a five-year period, from several provinces within SA.

\section{Study design}

\subsection{Specimen collection and processing}

From April 2009 to December 2013, 296 out of 477 SaV-positive specimens were referred from the Centre for Enteric Diseases, National Institute for Communicable Diseases, for further genotypic characterisation. As part of the ongoing Rotavirus Sentinel Surveillance Programme (RSSP), all specimens were screened for SaV and other enteric viruses [21]. Specimens were collected from children younger than six years old who were hospitalised with gastroenteritis in four provinces of SA (GP; KwaZulu Natal, KZN; Mpumalanga, MP and the Western Cape, WC). In addition, SaV-positive specimens were also received from a hospital on the GP-North West border (DGM) for characterisation of the strains present. The SaV-positive study population had a median age of 10 months and gender ratio of 1:0.66 male:female. All specimens were stored at $4^{\circ} \mathrm{C}$ until processing. Stool suspensions were prepared as approximate $10 \%$ weight/volume in sterile water.

\subsection{Nucleic acid extraction and reverse transcription}

Total nucleic acid was extracted from $200 \mu$ stool suspension using the MagNA Pure LC Total Nucleic Acid Isolation kit (Roche Diagnostics $\mathrm{GmbH}$, Mannheim, Germany) on the automated MagNA Pure platform (Roche Diagnostics) or from $160 \mu$ stool suspension using the Qiagen Viral RNA Mini Kit (Qiagen, Hilden, Germany) on the automated QIAcube platform (Qiagen). Reverse transcription was performed as previously described [24]. 


\subsection{Amplification of SaV partial capsid gene}

Approximately $300 \mathrm{bp}$ of a partial 5'-region of the SaV capsid gene was amplified for characterisation. The region was amplified by nested polymerase chain reaction (PCR) using published primers $[22,23]$ as previously described [24], with minor adjustments. Briefly, in the first round of PCR $5 \mu$ l cDNA was added to a $50 \mu \mathrm{l}$ reaction with $0.4 \mu \mathrm{M}$ of each primer (SV-F13, SV-F14, SV-DS3 and SV-DS4) and $1.25 \mathrm{U}$ AmpliTaq Gold DNA polymerase (Applied Biosystems, Foster City, CA), with cycling parameters as previously described [24]. The second round of PCR was performed in a $50 \mu$ reaction containing $2 \mu l$ of product from the first round of PCR, $0.4 \mu \mathrm{M}$ of each primer (SaV1245Rfwd, SV-DS5 and SV-DS6) and $1.25 \cup$ AmpliTaq Gold DNA polymerase. Amplicons were visualised under UV light following electrophoresis through a $1.5 \%$ agarose gel and staining with ethidium bromide. If no bands of the correct size (430 bp) were present, PCR was repeated with a different primer set (SaV124F, SaV1F, SaV5F, SV-R13 and SV-R14 for the first PCR and SaV1245Rfwd and SV-R2 for the second PCR) and the same cycling conditions to yield a $340 \mathrm{bp}$ amplicon.

\subsection{Sequencing and phylogenetic analysis}

Sapovirus amplicons were directly sequenced in both directions using the ABI PRISM BigDye ${ }^{\circledR}$ Terminator v. 3.1 Cycle sequencing kit (Applied Biosystems) on an ABI 3130 automated analyser (Applied Biosystems). M13(-21) and M13-Rev primer sequences were added to the 5'-end of the genotyping primers to facilitate sequencing. Nucleotide sequences were edited and analysed using Sequencher ${ }^{\text {TM }} 4.9$ (Gene Codes Corporation, Ann Arbor, MI) and BioEdit Sequence Alignment Editor (V.7.0.9.0) [25]. BLAST-n was used to determine the most closely related nucleotide sequences in GenBank [26]. Sequences were aligned with reference SaV strains, selected according to Oka et al. [13], using MAFFT Version 6 (https://align.bmr.kyushuu.ac.jp/mafft/online/server/). Phylogenetic analysis was performed in MEGA6 [27] using the neighbour-joining method with 1000 bootstrap replicates. Genotypes were assigned based on clustering with reference strains in the phylogenetic tree. Nucleotide sequences were submitted to GenBank: KC962463-510 and KP196379-566. 


\subsection{Statistical analysis}

Descriptive statistics were analysed using STATA12 software. Chi-square test was used to determine statistical significance ( $p$ values $\leq 0.05)$.

\section{Results}

From April 2009 to December 2013, 221 SaV strains were characterised from 296 SaV-positive specimens. The median age of the children from whom SaVs strains were genotyped was 11 months (Interquartile range: 7.2-16.5 months) and the male:female ratio was 1:0.65. Genotypes were assigned based on clustering with reference strains in the phylogenetic tree (Fig.1A) and 14 different SaV genotypes were identified from the 221 characterised strains (Table 1).

The genetic distribution of SaVs was not consistent for the different provinces in SA and the number of detected genotypes varied per province (Fig. 2). Thirteen genotypes were identified in GP, 12 in WC, 11 in KZN and eight in MP. Seven (GI.1, GI.2, GI.3, GII.1, GII.2, GI.4 and GIV) of the 14 genotypes were detected in all four provinces. KwaZulu Natal had the most varied genetic distribution when compared to the other provinces. Genotype II.1 predominated (25\%), followed by GII.4 (19\%) and GIV (18\%). This differed from the other provinces where GIV was one of the two most frequently identified genotypes. The two predominant genotypes in KZN, GII.1 and GII.4, were significantly more frequently reported in KZN than WC (11\% for GII.1; $6 \%$ for GII.4), the province with the second most frequent occurrence of these particular genotypes ( $p=0.0368$ for GII.1; $p=0.0156$ for GII.4). These significant differences emphasise the different genotypic profile for SaV in KZN when compared to the other provinces.

Other enteric viruses were present in $43 \%$ (95/221) of SaV-positive specimens, the majority (76\%) of which were co-infected with one other virus. Co-infections included adenovirus (AdV), astrovirus, bocavirus, norovirus (NoV) GI, NoV GII or rotavirus (RV) [21]. The most frequent co-infection was AdV (43\%), followed by NoV GII and RV in $21 \%$ of specimens each. There was no significant correlation between the SaV genotype identified in a specimen and the presence of multiple viruses in that specimen $(p=0.3778)$. 
Table 1. Sapovirus genotypes identified in children hospitalised with gastroenteritis from 2009 to 2013 in South Africa.

\section{No. of strains (\%)}

\begin{tabular}{|c|c|c|c|c|c|c|}
\hline Genotype & 2009 & 2010 & 2011 & 2012 & 2013 & Total \\
\hline GIV & $3(27)$ & $5(25)$ & $4(15)$ & $13(28)$ & $28(24)$ & $53(24)$ \\
\hline GI.2 & $1(9)$ & $4(20)$ & $10(37)$ & $2(4)$ & $23(20)$ & $40(18)$ \\
\hline Gl.1 & $3(27)$ & $1(5)$ & $2(7)$ & $7(15)$ & $17(15)$ & $30(14)$ \\
\hline Gll.1 & $1(9)$ & $2(10)$ & $1(4)$ & $9(20)$ & $14(12)$ & $27(12)$ \\
\hline GII.4 & $1(9)$ & & & $1(2)$ & $17(15)$ & $19(9)$ \\
\hline GII.3 & & & $3(11)$ & $8(17)$ & $3(3)$ & $14(6)$ \\
\hline GII.5 & & $3(15)$ & $2(7)$ & $2(4)$ & $1(1)$ & $8(4)$ \\
\hline GI.5 & & & $1(4)$ & $2(4)$ & $3(3)$ & $6(3)$ \\
\hline GI.3 & $1(9)$ & & $1(4)$ & $1(2)$ & $3(3)$ & $6(3)$ \\
\hline GII.2 & & $1(5)$ & $2(7)$ & $1(2)$ & $2(2)$ & $6(3)$ \\
\hline GI.6 & & $3(15)$ & $1(4)$ & & & $4(2)$ \\
\hline GI.7 & $1(9)$ & $1(5)$ & & & $2(2)$ & $4(2)$ \\
\hline GII.6 & & & & & $2(2)$ & $2(1)$ \\
\hline GII.7 & & & & & $2(2)$ & $2(1)$ \\
\hline Total & 11 & 20 & 27 & 46 & 117 & 221 \\
\hline
\end{tabular}




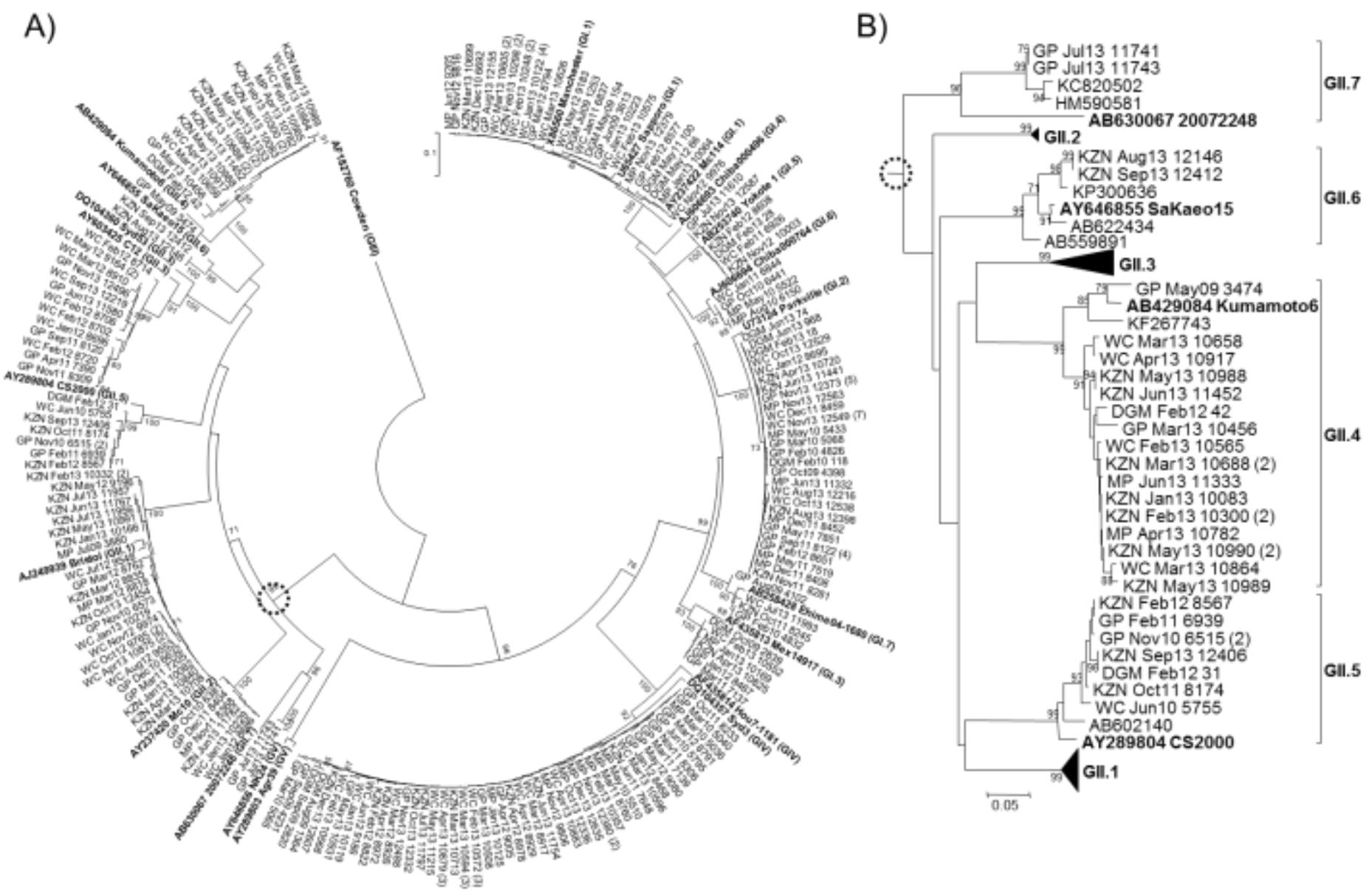

Figure 1A) Phylogenetic analysis of the partial capsid gene of sapoviruses (SaVs) detected in South Africa and B) an enlargement of the SaV genogroup II section of the tree. Neighbour-joining phylogenetic analysis of the partial capsid region (approximately $300 \mathrm{bp}$ ) of $221 \mathrm{SaV}$ strains. The SaV reference strains are indicated by GenBank accession numbers and are shown in bold. The tree was condensed by removing strains from the study that clustered together and originated from the same province and in the same month of the same year. The number of strains was then indicated in brackets next to the representative strain. Statistical significance was evaluated with a 1000 bootstrap replicates and bootstrap support of $>70 \%$ is indicated. The scale bar represents nucleotide substitutions per site. 


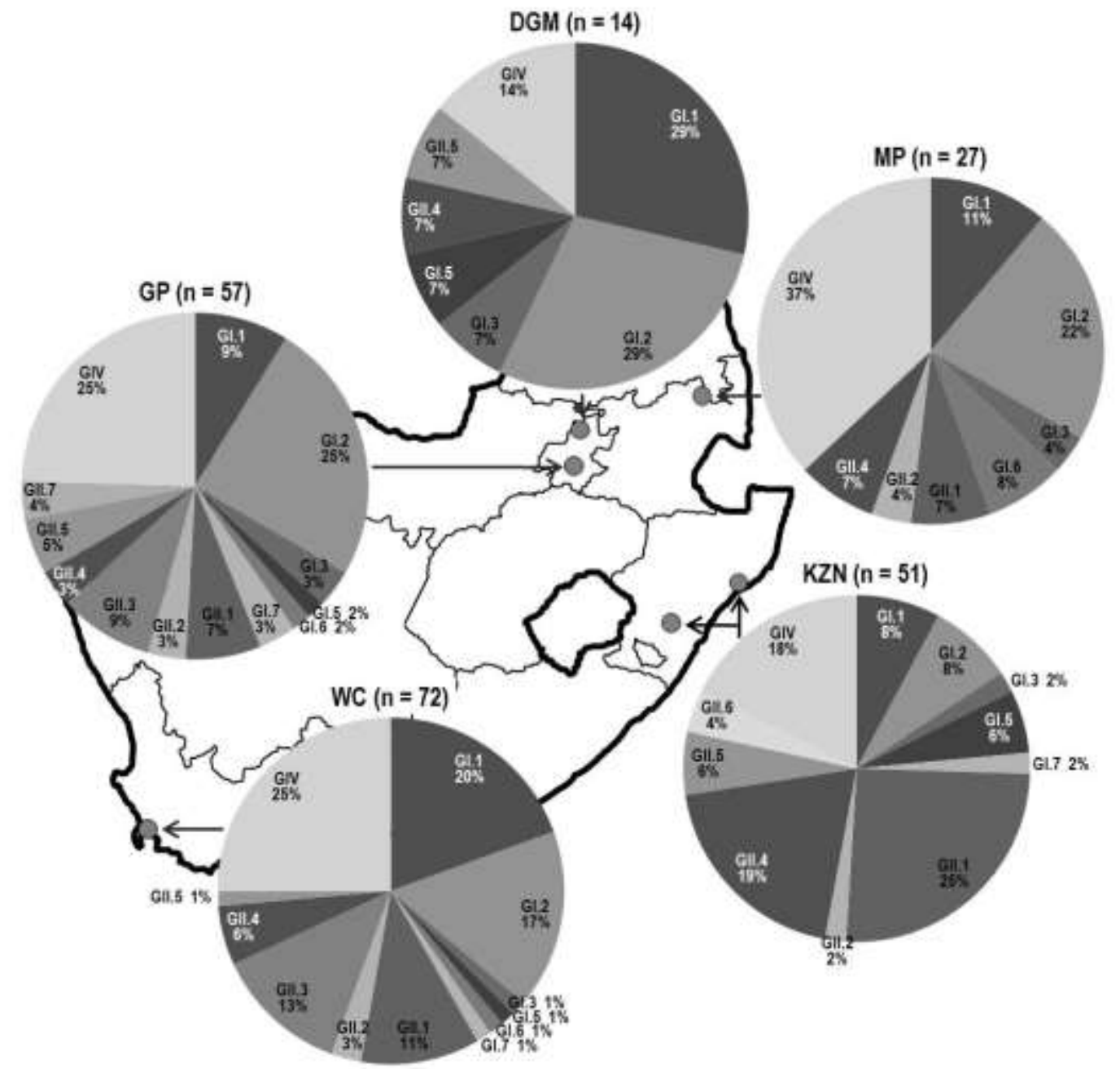

Figure $2 \mathrm{~A}$ Map indicating the provincial distribution of SaV genotypes within South Africa. Each grey circle represents a specimen collection site within a province (GP, Gauteng; MP, Mpumalanga; KZN, KwaZulu Natal; WC, Western Cape). Specimens from DGM (a site on the GP-North West province border) were not routinely available. However, those that were available and were SaV-positive were genotyped. The number of genotyped strains $(n)$ is indicated next to the province/collection site name. 
Table 2

Sapovirus genotypes detected in South Africa and their closest matches from other countries.

\begin{tabular}{|c|c|c|c|c|}
\hline \multirow[b]{2}{*}{ Genotype } & \multicolumn{4}{|c|}{ Closest GenBank matches } \\
\hline & \% Identity ${ }^{\mathrm{a}}$ & Year & Country & Accession number \\
\hline \multirow[t]{7}{*}{ GI.1 } & $98-100 \%$ & 2005 & Denmark & GQ340699 \\
\hline & & & Nicaragua & JX029963 \\
\hline & & 2006 & Russia & FJ214052 \\
\hline & & 2007 & Thailand & KC608715 \\
\hline & & 2008 & United Kingdom & FJ986146 \\
\hline & & 2010 & Brazil & KF924389 \\
\hline & & & Burkina Faso & КР300617 \\
\hline \multirow[t]{5}{*}{ GI.2 } & $99-100 \%$ & 2006 & Russia & FJ214045 \\
\hline & & 2008 & Iran & GU376748 \\
\hline & & 2010 & Brazil & KF924388 \\
\hline & & 2012 & China & KF974441 \\
\hline & & & Japan & AB894245 \\
\hline \multirow[t]{2}{*}{ GI.3 } & $99 \%$ & 2009 & Japan & AB622459 \\
\hline & & & Japan & AB689815 \\
\hline \multirow[t]{3}{*}{ GI.5 } & $98-99 \%$ & 2001 & Thailand & EU363881 \\
\hline & & 2003 & Russia & AY538715 \\
\hline & & 2005 & Japan & AB429094 \\
\hline \multirow{3}{*}{ GI.6 } & $99 \%$ & 2003 & Russia & AY538717 \\
\hline & & & Vietnam & DQ372736 \\
\hline & & 2008 & Japan & AB622435 \\
\hline \multirow[t]{3}{*}{ GI.7 } & $97-99 \%$ & 1990 & Pakistan & AB181133 \\
\hline & & 2001 & Thailand & EU363885 \\
\hline & & 2004 & Japan & AB258428 \\
\hline \multirow{5}{*}{ GII.1 } & $96-99 \%$ & 2005 & Nicaragua & JX162645 \\
\hline & & 2006 & Denmark & GQ340722 \\
\hline & & 2008 & United Kingdom & FJ986149 \\
\hline & & & Vietnam & JF262614 \\
\hline & & 2010 & China & KF495122 \\
\hline \multirow[t]{5}{*}{ GII.2 } & $98-99 \%$ & 2000 & Thailand & EU363877 \\
\hline & & 2001 & Australia & DQ104362 \\
\hline & & 2003 & United States & HM800904 \\
\hline & & 2006 & Denmark & GQ340710 \\
\hline & & 2008 & Japan & AB622433 \\
\hline \multirow[t]{2}{*}{ GII.3 } & $95-99 \%$ & 2010 & Japan & AB622456 \\
\hline & & & Nicaragua & KF361382-5 \\
\hline \multirow[t]{2}{*}{ GII.4 } & $90-94 \%$ & 2000 & Kenya & KF267743 \\
\hline & & 2003 & Japan & AB429084 \\
\hline GII.5 & $93-95 \%$ & 2005 & Japan & $\mathrm{AB} 602140^{\mathrm{b}}$ \\
\hline \multirow[t]{4}{*}{ GII.6 } & $91-97 \%$ & 2004 & Thailand & AY646855 \\
\hline & & 2008 & Japan & AB622434 \\
\hline & & & Spain & AB559891 ${ }^{\mathrm{b}}$ \\
\hline & & 2009 & Burkina Faso & КР300636 \\
\hline \multirow{2}{*}{ GII.7 } & $97-98 \%$ & 2010 & United States & HM590581 \\
\hline & & 2011 & China & КС820500 \\
\hline \multirow[t]{6}{*}{ GIV } & $96-99 \%$ & 2003 & Venezuela & HM214146 \\
\hline & & 2004 & Australia & DQ104357 \\
\hline & & 2007 & United States & HM800912 \\
\hline & & 2008 & United Kingdom & FJ986151 \\
\hline & & 2010 & Burkina Faso & KР300626 \\
\hline & & 2012 & China & KF649156 \\
\hline
\end{tabular}

a Denotes the range of percentages at which different South African strains identified in this study, within a specified genotype, are identical to the listed GenBank matches.

b Environmental samples, all other SaVs were detected in stool specimens. 
The predominant genotype identified in this study was GIV, comprising $24 \%$ of the genotyped strains and was followed by GI.2 at 18\% (Table 1). Many of the SaV strains from SA were closely related to SaVs identified elsewhere in the world, with nucleotide identities ranging from $90 \%$ to $100 \%$ over the typed region (Table 2). Within GI, SA strains were $97-100 \%$ identical over the genotyped region to the closest matches from GenBank. Genogroup IV strains from SA were also highly identical (96-99\%) to SaVs from other countries on several continents. More diversity was seen in GII where nucleotide identities ranged from 90-99\% between the SA SaVs and the closest GenBank matches. Within GII (GII.4, GII.5, GII.6 and GII.7), SA strains grouped separately from the closest SaVs from other countries (Fig. 1B). Sapoviruses from SA were not associated with the most closely related GenBank strains in terms of country of origin or time period of occurrence.

\section{Discussion}

This is the first report to provide comprehensive data on the genetic diversity of SaVs circulating in a paediatric population hospitalised with gastroenteritis in southern Africa. The study was conducted over a five-year period and includes specimens from several provinces within SA. A total of 221 of the 477 SaVpositive stool specimens were successfully genotyped. The median age and gender ratios of the SaVpositive population and the sub-population from which SaVs were characterised were highly similar. This suggests that the population from which SaVs were successfully genotyped is an adequate representation of the overall SaV-positive population. A notable diversity of SaVs was observed in the study, including 14 different genotypes. Currently 16 SaV genotypes are known to infect humans and GI.4 and GV were the only two that were not identified. Other studies have reported lower SaV genetic diversity [2,4,28-31], including a nine-year (2002-2011) study from Japan in which 11 genotypes were identified from 139 specimens [2] and a two-year (2005-2007) study from Denmark during which seven genotypes were characterised from 91 specimens [4]. The five-year time period of the study and the four different provinces in which specimens were collected are both likely to have influenced the genetic diversity seen in this study. This emphasises the need to conduct long term studies, preferably including more than one study site within a country. It is also possible that a higher genetic diversity of SaVs is circulating in children with gastroenteritis on the African continent. A recent study from Burkina Faso 
reported nine different genotypes characterised over an 11-month period [14] and a previous study from Kenya reported five genotypes from six specimens [18].

A wide genetic distribution of SaVs was observed in each of the four provinces. The number of genotypes ranged from eight in MP to 13 in GP. The number of detected genotypes is likely influenced by the number of strains characterised from a particular province, which varied greatly from 27 strains (MP) to 72 strains (WC). The genotypic distribution of SaVs in KZN differed significantly from other provinces. In KZN specimens were collected from a combination of rural and urban sampling sites whereas in the other provinces, the collection sites were either urban (GP, WC) or rural (MP).

Sapovirus single infections were present in $57 \%$ of the specimens from which SaVs were genotyped. Adenovirus was the most frequent co-infection and the possibility of a respiratory AdV co-infection cannot be excluded. Mixed infections, involving SaVs and other enteric viruses such as RV, AdV and NoV, at frequencies ranging from $17-26 \%$ have been reported previously [17, 30, 33].

The predominant genotype detected in children hospitalised with gastroenteritis in SA over the five-year period, GIV, was identified in each year and in all four provinces. The GIV strains from SA shared high nucleotide identity (96-99\%) with SaVs identified in patients with gastroenteritis in several countries over the past ten years, including Burkina Faso [14], China [10], the United Kingdom (UK), the United States (US) [9] and Venezuela [33]. This confirms a worldwide distribution of the genotype as well as indicating its presence in southern Africa. It also suggests stability of the genotype as older strains from 2003 shared high nucleotide identities over the partial capsid gene region to strains as recent as 2012 and 2013. Genogroup IV has been frequently reported in children as well as adults with gastroenteritis $[4,10,32,33]$ and particularly in SaV-associated outbreaks $[7,8,34,35]$. The second most prevalent and widely-distributed genotype, GI.2, is also frequently reported in patients with gastroenteritis worldwide $[6,8,10,30,31]$. Strains from SA shared even higher nucleotide identity (99-100\%) with SaVs from other countries, particularly recent strains reported in Asia (2012) and earlier strains from Brazil, Iran and Russia (2006-2010). 
Genotype II.1 was the most frequently detected GII genotype in SA. The SA strains shared high nucleotide identity (96-99\%) with strains from Europe, Asia and South America (2005-2010) [2, 31, 36]. Genotype II.2 strains from SA were also closely-related ( $98-99 \%$ nucleotide identity) to SaVs from various countries from 2000 to 2008 [2, 9, 29], whereas the GII.3 strains matched closely (95-99\% nucleotide identity) to SaVs recently detected in Japan (2010) and Nicaragua (2010) [31] only. The GII.4 SA strains shared $90-94 \%$ nucleotide identity with the two closest SaVs, older strains originating from Kenya (2000) [18] and Japan (2003) [5]. In GII.5, the most closely-related SaV (93-95\% nucleotide identity) was identified from wastewater in Japan (2005) [22] and in GII.6 GenBank matches with < 90\% nucleotide identity over the genotyped region included SaVs detected in stool from Burkina Faso [14], Thailand [37] and Japan and river water in Spain [23], from 2004 to 2009. In GII.7, closely-related SaVs from other countries included recent strains from the US (2010) and China (2011) [38]. Genotypes II.6 and II.7 were rarely found in SA, both emerging in 2013 only and each in a single province.

In SA, recent studies on the presence of SaVs in the environment have indicated a diversity of circulating genotypes which correlates to what was found in the clinical setting of this study. Twelve of the $14 \mathrm{SaV}$ genotypes identified in this study have been identified in various environmental samples in SA [24,39]. The strains circulating in the environment are highly identical to those detected in this clinical setting, with nucleotide identities ranging from $95-100 \%$. Interestingly, SaV GIV has not been reported in surface water [39] or effluent wastewater [24] in SA and was only recently identified in one water sample collected from an irrigation pivot on a fresh produce farm in SA [40]. The differing prevalence of SaV GIV in children with gastroenteritis and in the environment highlights the need to use passive environmental surveillance in combination with clinical studies to gain an accurate understanding of the genetic diversity of the virus in SA.

This study has provided new and comprehensive data on the genetic diversity of SaVs present in a population of children with gastroenteritis. Importantly, these results reflect the diversity of SaVs in severe gastroenteritis as the children were all hospitalised with the illness. The results provide valuable 
insight on the high diversity of SaV genotypes circulating in this population and the close genetic relatedness of GIV and GI SaV genotypes to strains circulating in many different countries on several continents worldwide. The study has also shown a more varied genetic relationship between GII SaVs from SA and those reported worldwide. The study has provided recent data on SaV genotypes in Africa, where data is lacking.

\section{Acknowledgements}

The authors would like to thank Dr J Mans for her input and suggestions for the manuscript. The authors acknowledge the contributions of the staff of the RSSP including principal investigators, surveillance officers, site supervisors, laboratory staff, data staff and study participants.

\subsection{Funding}

This study was partially supported by the South African Poliomyelitis Research Foundation (M.B.T. and T.Y.M.) and the National Research Foundation (NRF), SA (M.B.T., Grant specific reference number [UID 85799]). The Grantholder acknowledges that opinions, findings and conclusions or recommendations expressed in any publication generated by NRF supported research are that of the author(s), and that the NRF accepts no liability whatsoever in this regard. The RSSP is funded through a grant from GlaxoSmithKline.

\subsection{Competing interests}

None declared

\subsection{Ethical Approval}

Ethical approval for the RSSP was obtained from research ethics committees of the University of the Witwatersrand (M091018), University of KZN (BF074/09), University of Cape Town (068/2010) and University of Limpopo (MREC/P/10/2009). 


\section{References}

1. Oka T, Wang Q, Katayama K, Saif LJ. Comprehensive review of human sapoviruses. Clin Microbiol Rev 2015;28,32-53.

2. Johnsen CK, Midgley S, Böttiger B. Genetic diversity of sapovirus infections in Danish children 20052007. J Clin Virol 2009;46,265-9.

3. Miyoshi M, Yoshizumi S, Kanda N, Karino T, Nagano H, Kudo S, et al. Different genotypic sapoviruses detected in two simultaneous outbreaks of gastroenteritis among schoolchildren in the same school district in Hokkaido, Japan. Jpn J Infect Dis 2010;63,75-8.

4. Harada S, Oka T, Tokuoka E, Kiyota N, Nishimura K, Shimada Y, et al. A confirmation of sapovirus reinfection gastroenteritis cases with different genogroups and genetic shifts in the evolving sapovirus genotypes, 2002-2011. Arch Virol 2012;157,1999-2003.

5. Hassan-Rios E, Torres P, Munoz E, Matos C, Hall AJ, Gregoricus N, et al. Sapovirus gastroenteritis in preschool center, Puerto Rico, 2011. Emerg Infect Dis 2013;19,174-5.

6. Chhabra P, Samoilovich E, Yermalovich M, Chernyshova L, Gheorghita S, Cojocaru R, et al. Viral gastroenteritis in rotavirus negative hospitalized children $<5$ years of age from the independent states of the former Soviet Union. Infect Genet Evol 2014;28,283-8.

7. Mikula C, Springer B, Reichart S, Bierbacher K, Lichtenschopf A, Hoehne M. Sapovirus in adults in rehabilitation center, upper Austria. Emerg Infect Dis 2010;16,1186-7.

8. Svraka S, Vennema H, van der Veer B, Hedlund KO, Thorhagen M, Siebenga J, et al. Epidemiology and genotype analysis of emerging sapovirus-associated infections across Europe. $\mathrm{J}$ Clin Microbiol 2010;48,2191-8.

9. Lee LE, Cebelinski EA, Fuller C, Keene WE, Smith K, Vinjé J, et al. Sapovirus outbreaks in long-term care facilities, Oregon and Minnesota, USA, 2002-2009. Emerg Infect Dis 2012;18,873-6.

10. Wang G, Shen Z, Qian F, Li Y, Yuan Z, Zhang J. Genetic diversity of sapovirus in non-hospitalized adults with sporadic cases of acute gastroenteritis in Shanghai, China. J Clin Virol 2014;59,250-4.

11. Green K. Caliciviridae: The Noroviruses. In: Knipe DM, Howley PM, editors. Field's Virology, Vol. 1. Philadelphia: Lippincott Williams \& Wilkins; 2013, p. 582-608.

12. Farkas T, Zhong WM, Jing Y, Huang PW, Espinosa SM, Martinez N, et al. Genetic diversity among sapoviruses. Arch Virol 2004;149,1309-23.

13. Oka T, Mori K, Iritani N, Harada S, Ueki Y, lizuka S, et al. Human sapovirus classification based on complete capsid nucleotide sequences. Arch Virol 2012;157,349-52.

14. Matussek A, Dienus O, Djeneba O, Simpore J, Nitiema L, Nordgren J. Molecular characterization and genetic susceptibility of sapovirus in children with diarrhea in Burkina Faso. Infect Genet Evol 2015;32,396-400.

15. Sdiri-Loulizi K, Hassine M, Gharbi-Khelifi H, Aouni Z, Chouchane S, Sakly N, et al. Molecular detection of genogroup I sapovirus in Tunisian children suffering from acute gastroenteritis. Virus Genes 2011;43,6-12. 
16. Liu J, Kibiki G, Maro V, Maro A, Kumburu H, Swai N, et al. Multiplex reverse transcription PCR Luminex assay for detection and quantitation of viral agents of gastroenteritis. J Clin Virol 2011;50,30813.

17. Dove W, Cunliffe NA, Gondwe JS, Broadhead RL, Molyneux ME, Nakagomi O, et al. Detection and characterization of human caliciviruses in hospitalized children with acute gastroenteritis in Blantyre, Malawi. J Med Virol 2005;77,522-7.

18. Mans J, Murray TY, Kiulia NM, Mwenda JM, Musoke RN, Taylor MB. Human caliciviruses detected in HIV-seropositive children in Kenya. J Med Virol 2014;86,75-81.

19. Wolfaardt M, Taylor MB, Booysen HF, Engelbrecht L, Grabow WO, Jiang X. Incidence of human calicivirus and rotavirus infection in patients with gastroenteritis in South Africa. J Med Virol 1997;51,2906.

20. Mans J, de Villiers JC, du Plessis NM, Avenant T, Taylor MB. Emerging norovirus GII.4 2008 variant detected in hospitalised paediatric patients in South Africa. J Clin Virol 2010;49,258-64.

21. Page NA, Groome M, Murray TY, Nadan S, Netshikweta R, Kuonza L, Taylor MB, et al. Sapovirus prevalence in children less than five years of age hospitalised for diarrhoeal disease in South Africa, 2009 to 2013. J Clin Virol Submitted.

22. Kitajima M, Oka T, Haramoto E, Katayama H, Takeda N, Katayama K, et al. Detection and genetic analysis of human sapoviruses in river water in Japan. Appl Environ Microbiol 2010;76,2461-7.

23. Sano D, Pérez-Sautu U, Guix S, Pintó RM, Miura T, Okabe S, et al. Quantification and genotyping of human sapoviruses in the Llobregat river catchment, Spain. Appl Environ Microbiol 2011;77,1111-4.

24. Murray TY, Mans J, Taylor MB. Human calicivirus diversity in wastewater in South Africa. J Appl Microbiol 2013;114,1843-53.

25. Hall TA. BioEdit: a user-friendly biological sequence alignment editor and analysis program for Windows 95/98/NT. Nucl Acids Symp Ser 1999;41,95-8.

26. Altschul SF, Madden TL, Schaffer AA, Zhang J, Zhang Z, Miller W, et al. Gapped BLAST and PSIBLAST: a new generation of protein database search programs. Nucleic Acids Res 1997;25,3389-402.

27. Tamura K, Stecher G, Peterson D, Filipski A, Kumar S. MEGA6: Molecular Evolutionary Genetics Analysis version 6.0. Mol Biol Evol 2013;30,2725-9.

28. Gallimore $\mathrm{Cl}$, Iturriza-Gomara $\mathrm{M}$, Lewis D, Cubitt D, Cotterill H, Gray JJ. Characterization of sapoviruses collected in the United Kingdom from 1989 to 2004. J Med Virol 2006;78,673-82.

29. Malasao R, Maneekarn N, Khamrin P, Pantip C, Tonusin S, Ushijima H, et al. Genetic diversity of norovirus, sapovirus, and astrovirus isolated from children hospitalized with acute gastroenteritis in Chiang Mai, Thailand. J Med Virol 2008;80,1749-55.

30. Chhabra P, Payne DC, Szilagyi PG, Edwards KM, Staat MA, Shirley SH, et al. Etiology of viral gastroenteritis in children $<5$ years of age in the United States, 2008-2009. J Infect Dis 2013;208,790800.

31. Bucardo F, Reyes Y, Svensson L, Nordgren J. Predominance of norovirus and sapovirus in Nicaragua after implementation of universal rotavirus vaccination. PLoS One 2014;9,e98201. 
32. Ootsuka Y, Yamashita Y, Ichikawa T, Kondo R, Oseto M, Katayama K, et al. Molecular characterization of sapoviruses detected in sporadic gastroenteritis cases in 2007 in Ehime Prefecture, Japan. Jpn J Infect Dis 2009;62,246-8.

33. Gonzalez GG, Liprandi F, Ludert JE. Molecular epidemiology of enteric viruses in children with sporadic gastroenteritis in Valencia, Venezuela. J Med Virol 2011;83,1972-82.

34. Pang XL, Lee BE, Tyrrell GJ, Preiksaitis JK. Epidemiology and genotype analysis of sapovirus associated with gastroenteritis outbreaks in Alberta, Canada: 2004-2007. J Infect Dis 2009;199,547-51.

35. Yoshida T, Kasuo S, Azegami Y, Uchiyama Y, Satsumabayashi K, Shiraishi T, et al. Characterization of sapoviruses detected in gastroenteritis outbreaks and identification of asymptomatic adults with high viral load. J Clin Virol 2009;45,67-71.

36. Trang NV, Luan le T, Kim-Anh le T, Hau VT, Nhung le TH, Phasuk P, et al. Detection and molecular characterization of noroviruses and sapoviruses in children admitted to hospital with acute gastroenteritis in Vietnam. J Med Virol 2012;84,290-297.

37 Guntapong R, Hansman GS, Oka T, Ogawa S, Kageyama T, Pongsuwanna Y, et al. Norovirus and sapovirus infections in Thailand. Jpn J Infect Dis 2004;57,276-278.

38 Wu W, Yang H, Zhang HL, Xian HX, Yao XJ, Zhao DJ, et al. Surveillance of pathogens causing gastroenteritis and characterization of norovirus and sapovirus strains in Shenzhen, China, during 2011. Arch Virol. 2014;159,1995-2002.

39. Murray TY, Mans J, Taylor MB. First detection of human sapoviruses in river water in South Africa. Water Sci Technol 2013;67,2776-83.

40. Murray TY, Taylor MB. Quantification and molecular characterisation of human sapoviruses in water sources impacted by highly polluted discharged wastewater in South Africa. J Water Health In Press;doi:10.2166/wh.2015.255. 